\title{
Dependence of cell-free-layer width on rheological parameters: Combining empirical data on flow separation at microvascular bifurcations with geometrical considerations
}

\author{
Jakub Köry $\odot,{ }^{1, *}$ Philip K. Maini $\odot,{ }^{1}$ Joe M. Pitt-Francis $\odot,{ }^{2}$ and Helen M. Byrne ${ }^{1, \dagger}$ \\ ${ }^{1}$ Mathematical Institute, University of Oxford, Oxford OX2 6GG, United Kingdom \\ ${ }^{2}$ Department of Computer Science, University of Oxford, Oxford OX1 3QD, United Kingdom
}

(Received 6 September 2021; accepted 13 December 2021; published 24 January 2022)

\begin{abstract}
When blood flows through vessel networks, red blood cells (RBCs) are typically concentrated close to the vessel center line, creating a lubrication layer near the vessel wall. As RBCs bind oxygen, the width of this cell-free layer (CFL) impacts not only the blood rheology inside the vasculature, but also oxygen delivery to the tissues they perfuse and, hence, their function. Existing attempts to relate the width of the CFL to the rheological properties of the blood and the geometrical properties of the vessel are based on an analysis of the forces acting on RBCs suspended in the blood. However, the complexity of interactions in the blood makes this a challenging task. Here, we propose an alternative, two-step approach to derive such a functional relationship. First, we extend widely accepted empirical fits describing the minimum flow fraction needed for RBCs to enter a daughter vessel downstream of a microvascular bifurcation so that it depends not only on the diameter and discharge haematocrit of the parent vessel, but also on its average shear rate. Second, we propose a simple geometrical model for the minimum flow fraction based on the cross-sectional blood flow profile within the parent vessel upstream of the bifurcation-considering uniform, parabolic, and blunt velocity profiles—and derive the leading-order approximation to this model for small CFL widths. By equating the functional relationships obtained using these two approaches, we derive expressions relating the CFL width to the vessel diameter, discharge haematocrit, and mean shear rate. The resulting expressions are in good agreement with available in vivo data and represent a promising basis for future research.
\end{abstract}

DOI: 10.1103/PhysRevE.105.014414

\section{INTRODUCTION}

Healthy functioning of living tissues depends in many ways on an adequate supply of oxygen [1,2]. Moreover, an increased understanding of the mechanisms underlying oxygen delivery to living tissues is a prerequisite for successful anticancer treatment, as the efficacy of radiotherapy and chemotherapy is known to be highly sensitive to local oxygen levels [3-7]. To accurately predict tissue oxygenation, a detailed knowledge of red-blood-cell (RBC) distribution in the vascular network contained in the tissue is required. This distribution depends on the way in which RBCs partition at diverging bifurcations which, in turn, depends on various geometrical and rheological parameters. Moreover, even within the cross section of a single vessel, RBCs preferentially travel near the center line, creating a layer near the vessel wall lacking RBCs. This cell-free layer (CFL) acts as a lubrication layer and its width (sometimes also referred to as thickness) directly correlates with the flow resistance [8]. RBCs carry oxygen and, thus, act as a buffer modulating the balance between cell-bound and unbound (free, plasma) oxygen, with only the latter being available for extravasation and diffusion into the tissue in which the vessels are located.

The CFL originates from complex interactions involving the cellular constituents of the blood, plasma fluid, and vessel

\footnotetext{
*Also at Mathematics and Statistics, University of Glasgow, 132 University Place, Glasgow G12 8TA, United Kingdom.

†helen.byrne@maths.ox.ac.uk
}

wall. As summarized in [9], RBCs migrate towards the center line due to their interactions with the vessel wall (and, more importantly, the endothelial cell layer) under shear flow, and the curvature of the flow profile. They also move down density gradients, towards the vessel wall, due to intercellular interactions (collisions). Other effects, such as cell aggregation, are also likely to have a significant impact on the CFL width [8].

As observed in vivo [10,11] and studied extensively in silico using the dissipative particle dynamics (DPD) method [8,12-14], the width $W$ of a fully established CFL depends on a range of parameters, including the vessel discharge haematocrit (the volume fraction of RBCs in blood) $H_{D}$, the vessel diameter $D$, and the average shear rate $\overline{\dot{\gamma}}$. DPD simulations typically show that $W$ increases as $\bar{\gamma}$ and $D$ increase, and $H_{D}$ decreases (see Fig. 6 in Appendix A). Recently, immersed boundary lattice Boltzmann simulations of RBCs suspended in plasma were extended to account for platelet dynamics $[15,16]$ as well as the transport of solid particles in blood flow [17,18]. However, these simulations tend to be computationally expensive due to the complexity of the interactions they model. As such, they are often limited to studies of simple networks with few vessels. Moreover, explicit closed-form expressions relating the CFL width to rheological parameters are rare as there is no simple way to arrive at such reduced-order models from the detailed simulations. Such expressions are needed in order to improve the accuracy of the existing two-phase models of blood flow in a single vessel (e.g., [19]) that often assume the CFL width to be constant [20] and in order to facilitate modeling and 
simulation of larger networks. Efforts to derive such expressions typically balance the forces that push RBCs away from and towards the vessel wall. For example, in [8], the lift force driving RBCs away from the vessel wall is balanced by an effective pressure representing intercellular interactions.

\section{Motivation}

Blood rheology is extremely complex, and it is thus a challenge to appropriately account for all participating forces and derive a reliable functional relationship for the dependence of CFL width on rheological parameters. For instance, Fig. 6(b) demonstrates that even though the model for CFL width developed therein showed good agreement with DPD simulations using vessel diameters of $20 \mu \mathrm{m}$ after fitting two parameters, the agreement using $40 \mu \mathrm{m}$ for vessel diameters was much poorer. Moreover, detailed DPD simulations at the scale of an entire microvasculature are, to date, still computationally intractable. Thus, it is desirable to have a reliable yet simple functional relationship relating CFL width to key rheological parameters. In this paper, we derive such a relationship using a different approach: we combine insight gained from existing in vivo data which show how RBCs partition at diverging microvascular bifurcations with simple geometrical considerations. In this way, we build an important bridge between two well-studied subfields of vascular rheology-empirical models of RBC splitting at microvascular bifurcations and DPD simulations of RBCs suspended in plasma.

At diverging microvascular bifurcations, RBCs do not split in proportion to the respective flow rates of the two daughter vessels; typically, the daughter vessel with the higher flow rate receives disproportionately more RBCs. This phenomenon has been termed haematocrit splitting (HS) and is caused by interactions between multiple physical factors (including the presence of a CFL).

A number of mathematical models for HS have been proposed [21-27]. Pries and co-workers [21-23] developed what is arguably the most widely used phenomenological model for HS and fitted the parameters of their model to experimental data collected from arteriolar bifurcations in rat mesentery. One of the key parameters in their HS model is $X_{0}$, the minimum fractional flow rate required for RBCs to enter either daughter branch (i.e., if the fractional flow rate in one of the branches is less than $X_{0}$, then no RBCs enter this branch). The minimum fractional flow rate $X_{0}$ depends on the cross-sectional flow profiles of the RBCs and plasma flow in the (parent) vessel just upstream of the diverging bifurcation (see Fig. 1). The vessel cross section can be partitioned into two subregions by a separating curve (whose shape depends on many factors), so that all blood passing through the first subregion flows into the first branch and that passing through the second subregion flows into the second daughter branch. Thus, the minimum fractional flow rate corresponds to the threshold case where the CFL fully contains one of these subregions [see Fig. 2(a)].

In [22], the authors proposed an empirical expression for $X_{0}$ of the form

$$
X_{0}=\frac{0.4}{D}
$$

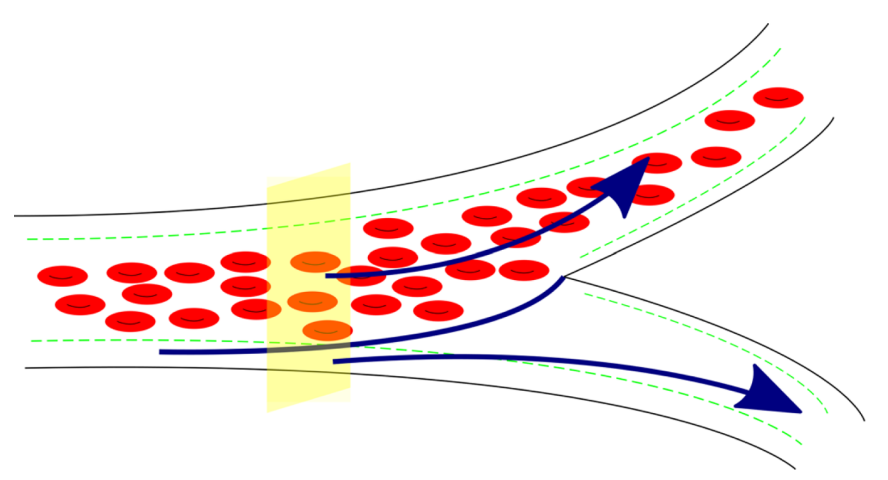

FIG. 1. The minimum fractional flow rate $X_{0}$ needed for RBCs to enter a daughter vessel depends on the flow and haematocrit profile in the vessel upstream of a diverging bifurcation. Key: the dashed green curves mark the edges of CFLs; the yellow parallelogram depicts a vessel cross section upstream of the bifurcation; the dark blue curves describe how the (plasma) flow separates at the bifurcation.

where $D$ denotes the parent vessel diameter. A subsequent refinement of this empirical fit was proposed in [23]; the modified fit accounts for the dependence of $X_{0}$ on the parent vessel discharge haematocrit $H_{D}$ (for more details, see Appendix B) and takes the form

$$
X_{0}=0.964\left(1-H_{D}\right) / D .
$$

Throughout this work, we will make use of the following simplifying assumptions (SAs):

SA.1: The flow in the parent vessel is fully established.

SA.2: The CFL width is independent of space and time.

SA.3: The vessel cross section is circular.

SA.4: The separating curve is a straight line segment.

SA.5: RBCs are uniformly distributed outside the CFL.

SA.6: The center of mass of every RBC follows the flow lines.

SA.7: The diameter of RBCs is negligible in comparison with the vessel diameter.

SA.8: The CFL width is much smaller than the vessel diameter.

We note that SA.1 implies that any effects of CFL disturbance and recovery associated with perturbations of the flow at upstream bifurcations can be neglected [27], while SA.2 implies that spatiotemporal variations in the $\mathrm{RBC}$ and plasma profiles can be neglected and that the CFL width should be interpreted as an averaged value.

Under these assumptions, $X_{0}$ will depend on the CFL width $W$ and the diameter $D$ of the parent vessel. Using simple geometrical arguments, we will derive such relationships for different flow profiles in the vessel cross section. By comparing the resulting relationships with the empirical fits defined by Eqs. (1) and (2), we will derive models that relate the CFL width $W$ to key rheological parameters.

The remainder of the paper is organized as follows. In Sec. II A, we first extend the empirical fits given by Eqs. (1) and (2) to account for the dependence of $X_{0}$, via $W$, on the average shear rate $\bar{\gamma}$ as observed in DPD simulations from [8]. In Sec. II B, we then derive functional relationships relating the minimum fractional flow rate $X_{0}$ to $W$ and $D$ based purely on geometrical ideas of flow and RBC separation. Comparing these geometrical models with the extended empirical fits 


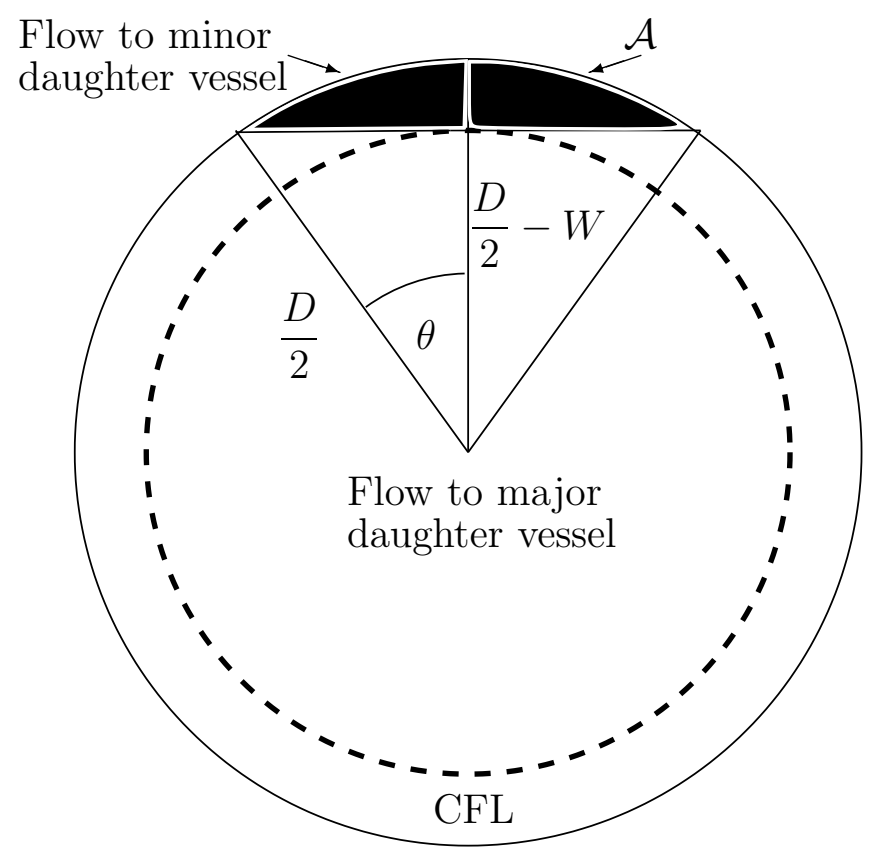

(a)

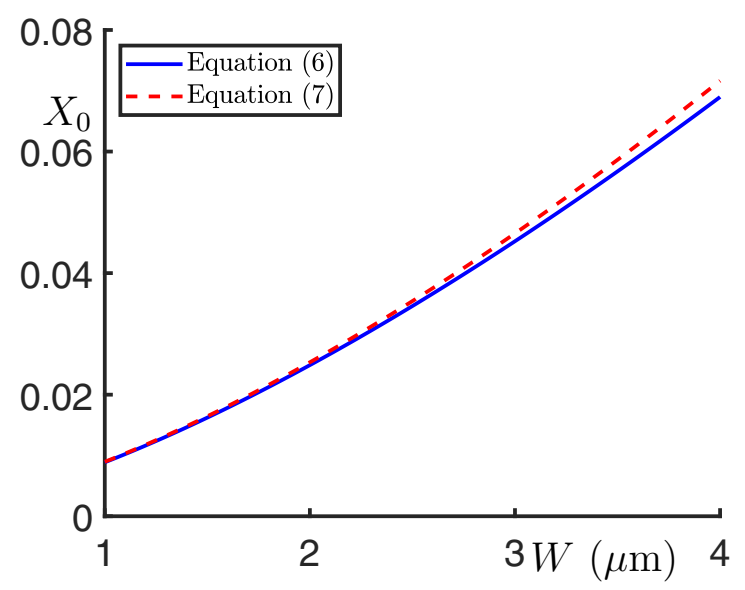

(b)

FIG. 2. Flow-separation considerations within the vessel cross section yield relationships between the CFL width $W$ and the minimum flow fraction $X_{0}$ (for a given parent vessel diameter) that depend on the functional form used to describe the velocity profile within a vessel cross section upstream from the bifurcation. Panel (b) shows $X_{0}$ as a function of $W$ for $D=33 \mu \mathrm{m}$. The red dashed curve represents the approximation (7) for small $W / D$, assuming a uniform velocity profile, and is in good agreement with the exact formula (6) (solid blue curve) for this range of values of $W$.

obtained in Sec. II A, we obtain implicit equations for how $W$ depends on $H_{D}, D$, and $\overline{\dot{\gamma}}$. Making use of Taylor expansions for the case $W / D \ll 1$, we can then invert the leading-order approximations to these equations, conclude explicit formulas for $W$, and compare the predictions of these formulas against some of the simulations from our previous work [27] (using a lattice Boltzmann method). We validate these models against in vivo CFL width measurements in Sec. III before concluding with a summary of the main results and a discussion of possible directions for future investigations in Sec. IV.

\section{DERIVATION OF A HYBRID MODEL FOR THE CFL WIDTH}

In this section, we develop simple models that relate the CFL width $W$ to the vessel discharge haematocrit $H_{D}$, vessel diameter $D$, and average shear rate $\overline{\dot{\gamma}}$, where $\bar{\gamma}=\bar{v} / D$ and $\bar{v}$ is the average flow velocity. To achieve this, we first extend existing empirical fits [21-23] so that $X_{0}$, the minimum flow fraction needed for RBCs to enter one of the daughter vessels at a diverging bifurcation, depends not only on the discharge haematocrit $H_{D}$ and diameter $D$ of the parent vessel, but also on the average shear rate $\bar{\gamma}$ (Sec. II A). We then use geometrical arguments to relate the minimum flow fraction $X_{0}$ to the CFL width $W$ for three different flow profiles in the parent vessel (uniform, parabolic, and blunt velocity profiles; see Sec. II B). By equating these expressions with the extended empirical fits from Sec. II A, we arrive at implicit expressions for $W$ which depend on key rheological parameters. Under the additional assumption that $W \ll D$, these expressions simplify to give explicit formulas for $W$ that are correct to leading order. For consistency with [21-23], in what follows, all length scales have been nondimensionalized so that their values signify their dimensional values expressed in microns.

\section{A. Dependence of the minimum flow fraction on the parent vessel diameter, haematocrit, and shear rate}

Experimental studies [21-23] introduced the two aforementioned functional forms given by Eqs. (1) and (2) relating the minimum flow fraction $X_{0}$ to the diameter $D$ and discharge haematocrit $H_{D}$ of the upstream parent vessel, in a physically plausible way: $X_{0}$ is inversely proportional to $D$ and decreases (linearly) with increasing $H_{D}$ (for details, see Appendix B). In practice, however, two vessels with similar values of $H_{D}$ and $D$ may experience different pressure gradients and, hence, different flow rates, velocity profiles, and shear rates. Given that (a fully established) CFL width depends strongly on the shear rate [8], we postulate that the minimum flow fraction $X_{0}$ should also depend on $\overline{\dot{\gamma}}$.

\section{Extension of Equation (2)}

We extend the empirical fit defined by Eq. (2) by replacing the constant factor 0.964 with a function $f=f(\dot{\gamma})$ of the average shear rate $\overline{\dot{\gamma}}$, interpreting this particular factor as a value averaged across a potential range of shear rates experienced in vascular networks from [21] (noting that [21] did not investigate how $X_{0}$ depends on $\overline{\dot{\gamma}}$ ). As discussed in [8], $W$ is an increasing function of $\bar{\gamma}$, which attains a finite value as $\bar{\gamma} \rightarrow \infty$ [see Figs. 6(b) and 6(c)]. Based on this observation, we propose the following functional form for $f(\bar{\gamma})$ :

$$
f(\overline{\dot{\gamma}})=C_{1} \times\left(1-e^{-C_{2} \bar{\gamma}}\right),
$$

where the constants $C_{1}$ and $C_{2}$ are chosen so that the model is consistent with the existing literature. The dimensionless parameter $C_{1}$ represents the maximum value of the function $f$, which is realized in the limit as $\bar{\gamma} \rightarrow \infty$ and estimated below. The dimensional parameter $C_{2}$ (in seconds) modulates the impact of the shear rate $\bar{\gamma}$ on the minimum flow fraction $X_{0}$ and, in turn, the CFL width $W$. It is estimated in Sec. II B. 
Unfortunately, we could not find any quantitative information on local shear rates, flow rates, and velocities in [21], which contains the experimental work that gave rise to Eq. (2). In order to estimate values of the mean shear rate, we exploited simulation results from [22] in which a model of blood flow through microvascular networks was developed and parameterized using data from [21]. The resulting predictions of the average flow velocity were in good agreement with experiments from a separate study [28]. Taking typical values of the flow velocity $\bar{v} \approx 1 \mathrm{~mm} \mathrm{~s}^{-1}$ and vessel diameter $D \approx$ $10 \mu \mathrm{m}$ from [28], we estimate $\bar{\gamma}=\bar{v} / D \approx 100 \mathrm{~s}^{-1}$. From Fig. 6(b), we note that for such values of $\bar{\gamma}$, the CFL width is close to its maximum value [corresponding to $\overline{\dot{\gamma}} \rightarrow \infty$ and, thus, $f \approx C_{1}$ in (3)]. For simplicity, and consistency with the constant factor of 0.964 that appears in Eq. (2), we set $C_{1}=1$ as the default value throughout this work and study the impact of uncertainty in this parameter in Sec. III (see Fig. 5).

In summary, we extend Eq. (2) to the following empirical fit, which incorporates the dependence of $X_{0}$ on the average shear rate:

$$
X_{0}\left(\bar{\gamma}, H_{D}, D\right)=\frac{C_{1}\left(1-e^{-C_{2} \bar{\gamma}}\right)\left(1-H_{D}\right)}{D} .
$$

\section{Extension of Equation (1)}

In a similar manner, we propose the following extension of Eq. (1), replacing the factor 0.4 with $g(\bar{\gamma})=C_{3} \times\left(1-e^{-C_{4} \bar{\gamma}}\right)$ (with $C_{3}=0.4$ being the default value) so that

$$
X_{0}(\overline{\dot{\gamma}}, D)=\frac{C_{3} \times\left(1-e^{-C_{4} \bar{\gamma}}\right)}{D} .
$$

As above, we postpone determination of the constant $C_{4}$ (in seconds) to the next section. We note that in practice, even when $\bar{\gamma}=0$ (i.e., no flow), a CFL with $W>0$ exists due to repulsive forces between $\mathrm{RBCs}$ and the tube wall, as discussed in [8]. However, the effects underlying this phenomenon significantly affect the CFL width only at very low shear rates [8] and will therefore be neglected here.

\section{B. Relating the CFL width and the minimum flow fraction}

In this section, we consider a (three-dimensional) parent vessel of diameter $D$, which splits into two daughter vessels, and focus on the two-dimensional cross section of this vessel upstream from, and in close proximity to, the bifurcation. We partition the cross section into two subdomains, with blood from the first (second) subdomain entering the first (second) daughter vessel. We use geometrical arguments to establish a functional relationship between the minimum flow fraction $X_{0}$ and the corresponding undisturbed CFL width $W$. We start by studying a highly idealized case for which the velocity profile is uniform across the vessel cross section. We then analyze the more realistic case of a parabolic velocity profile, resulting from the Poiseuille law governing Stokes flow in cylindrical channels. Finally, we consider a blunt velocity profile, representing the most realistic description of velocity profile observed in microvasculature (see [29], for instance). Where necessary, we use superscripts $U, P$, and $B$ to distinguish between the three cases and emphasize the assumptions associated with the uniform, parabolic, and blunt velocity profiles, respectively. The superscripts $L B$ and $I V$ indicate whether relevant CFL widths were obtained from lattice Boltzmann simulations [27] or in vivo data [10,11], respectively.

\section{Uniform velocity profile}

By definition, the minimum flow fraction $X_{0}$ can be equated with the black (cell-free) area $\mathcal{A}$ in Fig. 2(a), divided by the area of the whole cross section, $\pi(D / 2)^{2}$. Throughout this work, we will assume that errors resulting from the nonzero size of RBCs are negligible (see SA.7). Using Pythagoras' theorem, it is straightforward to show that the black area can be calculated as

$\mathcal{A}=\arccos \left(1-\frac{2 W}{D}\right)\left(\frac{D}{2}\right)^{2}-\left(\frac{D}{2}-W\right) \sqrt{W(D-W)}$,

and, hence, that

$$
X_{0}=\frac{\arccos \left(1-\frac{2 W}{D}\right)-2\left(1-\frac{2 W}{D}\right) \sqrt{\frac{W}{D}-\left(\frac{W}{D}\right)^{2}}}{\pi} .
$$

Inverting this relationship to obtain an explicit expression for $W=W\left(X_{0}\right)$ is not straightforward, even for fixed values of $D$. However, as the area of the black region in Fig. 2(a) is an increasing function of $W$, we note that $X_{0}$ is also an increasing function of $W$. Thus, given a fixed value of the diameter $D$, we can plot (6) and, for any value of $X_{0}$, read off the corresponding value of $W$ [see Fig. 2(b), where $D=33 \mu \mathrm{m}$, for consistency with the results from lattice Boltzmann simulations presented in [27], which are compared with our results below]. Under SA.8, we can obtain an approximate function for $X_{0}=X_{0}(W)$ which (assuming constant $D$ ) can be inverted to obtain $W=W\left(X_{0}\right)$. Defining $\delta=W / D$ to be the CFL width nondimensionalized with respect to the vessel diameter, we expand (6) in terms of $\delta \ll 1$ to obtain

$$
X_{0}^{U}=\frac{16}{3 \pi} \delta^{\frac{3}{2}}+O\left(\delta^{\frac{5}{2}}\right) \approx \frac{16}{3 \pi}\left(\frac{W^{U}}{D}\right)^{\frac{3}{2}},
$$

recalling that the superscript $U$ indicates that we are assuming that the velocity profile is uniform. This approximate expression is valid for CFL widths that are small compared to vessel diameter [compare the dashed red line and the solid black lines in Fig. 2(b)] and can be inverted to give

$$
W^{U} \approx D\left(\frac{3 \pi X_{0}^{U}}{16}\right)^{\frac{2}{3}}
$$

In order to estimate $C_{2}$ in Eq. (4) for a uniform velocity profile, we use the results from [8] for the lowest available value of $\bar{\gamma}$ [reproduced in Fig. 6(a)]. We observe that the value of an established CFL width for $\bar{\gamma}=9 \mathrm{~s}^{-1}$ (approximately $1.3 \mu \mathrm{m}$ in this case) is about 0.65 times the value for infinite $\bar{\gamma}$ (estimated to be $2 \mu \mathrm{m}$ from the same figure). Using Eqs. (4) and (7), we deduce

$$
\begin{aligned}
1-e^{-9 C_{2}^{U}} & =\frac{X_{0}^{U}\left(\bar{\gamma}=9 \mathrm{~s}^{-1}\right)}{X_{0}^{U}(\bar{\gamma}=\infty)} \\
& =0.65^{\frac{3}{2}} \approx 0.52 \Rightarrow C_{2}^{U} \approx 0.08 \mathrm{~s} .
\end{aligned}
$$

Using a similar argument, we can derive a similar estimate for $C_{4}$ in Eq. (5). Comparing the minimum flow fraction $X_{0}^{U}$ (for a fully established CFL) obtained using the above geometrical 


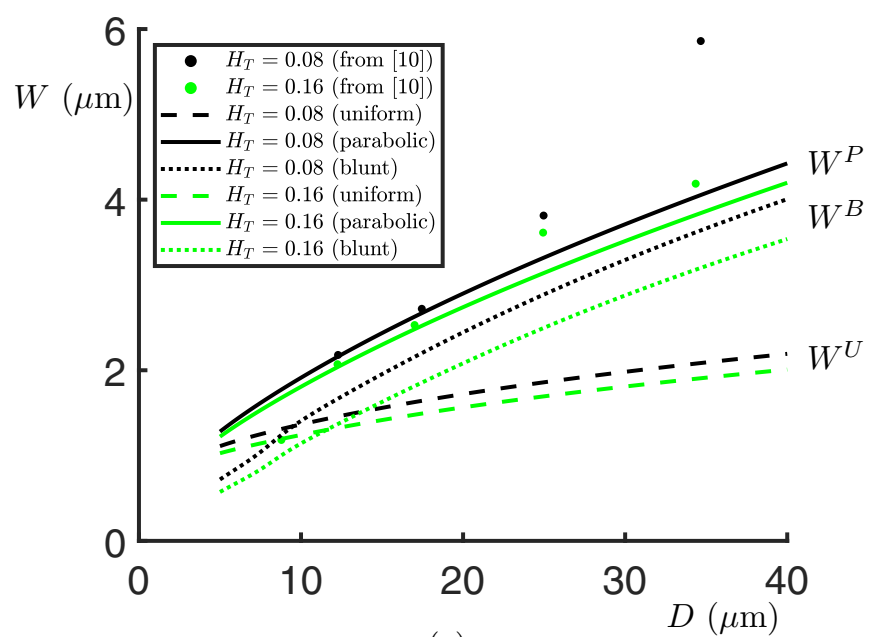

(a)

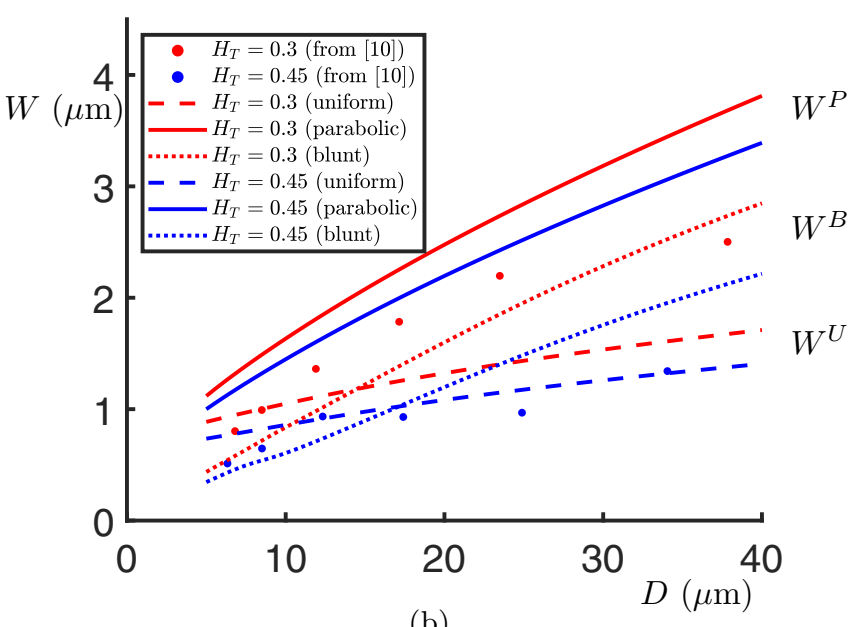

(b)

FIG. 3. Fully established CFL width $(W)$ as a function of vessel diameter $(D)$ for a range of values of the tube haematocrit. (a) Results for $H_{T}=0.08$ (black) and 0.16 (green); (b) those for $H_{T}=0.30$ (red) and 0.45 (blue). Data points represent in vivo measurements from [10], solid lines correspond to predictions from the parabolic model (17), dotted lines to the blunt-profile model (20), and dashed lines to the uniform-profile model (12). The parabolic model gives the most accurate predictions for low-haematocrit values (although for very low haematocrit- 0.08 - and large vessel diameters, the approximation breaks down). For higher-haematocrit values, the accuracy of the uniform-profile model increases.

arguments in the small-width limit [i.e., Eq. (7)] with the extended empirical fit defined by Eq. (4), we have

$$
\frac{C_{1}\left(1-e^{-0.08 \bar{\gamma}}\right)\left(1-H_{D}\right)}{D}=\frac{16}{3 \pi}\left(\frac{W^{U}}{D}\right)^{\frac{3}{2}}
$$

or, equivalently,

$$
W^{U}=\left(\frac{3 \pi C_{1}\left(1-e^{-0.08 \bar{\gamma}}\right)\left(1-H_{D}\right) D^{\frac{1}{2}}}{16}\right)^{\frac{2}{3}} .
$$

Recalling that in the empirical fits from [21-23], $D$ is (formally) dimensionless and vessel diameters are defined in microns, we note that for (10) and (11) to hold, $W^{U}$ must be
TABLE I. Key parameters used in the lattice Boltzmann simulations from [27] for the vessel downstream of the first bifurcation for which the CFL recovery was studied (vessel numbered as 1 in the reference).

\begin{tabular}{lll}
\hline \hline Parameter & \multicolumn{1}{c}{ Description } & \multicolumn{1}{c}{ Value } \\
\hline$D$ & vessel diameter & $33(\mu \mathrm{m})$ \\
$\bar{v}$ & average velocity & $300(\mu \mathrm{m} / \mathrm{s})$ \\
$H_{D}$ & haematocrit & $20 \%$ \\
\hline \hline
\end{tabular}

defined in the same way. Equation (11) can also be written as

$$
W^{U}=K_{1}\left(1-e^{-0.08 \bar{\gamma}}\right)^{\frac{2}{3}}\left(1-H_{D}\right)^{\frac{2}{3}} D^{\frac{1}{3}},
$$

where $K_{1}=\left(3 \pi C_{1} / 16\right)^{2 / 3} \approx 0.7$. We note that if we equate Eq. (7) with Eq. (5) instead of (4), then we obtain an expression for the CFL width which is independent of the discharge haematocrit, contradicting all available evidence (a similar result holds for the parabolic velocity profile). Therefore, henceforth, we consider only the extended empirical fit defined by Eq. (4).

We next compare (12) with the CFL width observed in lattice Boltzmann simulations from [27] where the (fully established) CFL width was estimated to be $W^{L B} \approx 1.9 \mu \mathrm{m}$ (see Figs. 3(f) and 3(g) in [27]). From Table I, it is straightforward to estimate the average shear rate in the simulations from [27], $\bar{\gamma}=\bar{v} / D \approx 9 \mathrm{~s}^{-1}$. Using values from Table I, Eq. (12) yields $W^{U} \approx 1.24 \mu \mathrm{m}$, which contrasts with a value $W^{L B} \approx 1.9 \mu \mathrm{m}$ obtained from the lattice Boltzmann simulations [27]. In practice, the assumption of a uniform velocity profile may not be valid for blood rheology, as the velocity $v$ should vanish on the vessel wall $(r=D / 2$, where $r$ denotes the polar radius). Under the assumption of a single-phase viscous fluid flowing through a cylindrical channel, we obtain the standard parabolic velocity profile. The presence of a second phase$\mathrm{RBCs}$ - causes the profile to be blunter than the parabolic one and, thus, one might anticipate good agreement with the uniform-profile model only for high haematocrit levels (see Fig. 7). Due to the combination of low discharge haematocrit and (relatively) large vessel diameter used in [27], we expect the value of the CFL width to be close to that predicted for a parabolic velocity profile. The aforementioned blunt velocity profiles can be viewed as intermediate between the uniform and the parabolic velocity profiles (see [12], for instance). Therefore, the CFL width (both in silico and in vivo) is expected to be intermediate between that for the uniform and parabolic velocity profiles. Finally, since the velocity should vanish on the vessel wall, if we assume a uniform velocity profile, then we overestimate the flux of blood that passes through the CFL. Equating the resulting expression for the minimum flow fraction $X_{0}^{U}$ with the empirical fit from [23] thus yields a model that systematically underestimates the CFL width.

\section{Parabolic velocity profile}

Under the assumption of (one-phase, i.e., plasma) steady laminar flow in a cylindrical vessel, the velocity profile 


$$
v^{P}=v^{P}(r) \text { satisfies }
$$

$$
v^{P}(r)=\frac{32 Q}{\pi D^{4}}\left[\left(\frac{D}{2}\right)^{2}-r^{2}\right]
$$

where $Q$ denotes the volumetric (blood) flow rate (for details, see [29]). The minimum flow fraction $X_{0}^{P}$ equals the flow rate through the black (cell-free) area $\mathcal{A}$ from Fig. 2(a), divided by $Q$, i.e.,

$$
\begin{aligned}
X_{0}^{P} & =\left\{\int_{D / 2-W}^{D / 2}\left[\int_{\arcsin \left(\frac{D / 2-W}{r}\right)}^{\pi-\arcsin \left(\frac{D / 2-W}{r}\right)} d \varphi\right] r v^{P}(r) d r\right\} / Q=\frac{32}{\pi D^{4}} \int_{D / 2-W}^{D / 2}\left(\frac{D^{2}}{4} r-r^{3}\right)\left[\pi-2 \arcsin \left(\frac{D / 2-W}{r}\right)\right] d r \\
& =\frac{16}{3 \pi D^{4}}\left\{-\frac{3 D^{3}}{8} \sqrt{(D-W) W}-\frac{D^{2}}{4} \sqrt{(D-W) W^{3}}+3 D \sqrt{(D-W) W^{5}}-2 \sqrt{(D-W) W^{7}}\right\}+\frac{1}{\pi} \arccos \left(1-\frac{2 W}{D}\right) .
\end{aligned}
$$

This, again, is difficult to invert but, under SA.8 and assuming $\delta \ll 1$, we can rewrite it and perform a Taylor series expansion to obtain the following, leading-order approximation:

$$
X_{0}^{P}=\frac{256}{15 \pi} \delta^{\frac{5}{2}}+O\left(\delta^{\frac{7}{2}}\right) \approx \frac{256}{15 \pi}\left(\frac{W^{P}}{D}\right)^{\frac{5}{2}} .
$$

The above expression can be inverted to give

$$
W^{P}=D\left(\frac{15 \pi X_{0}^{P}}{256}\right)^{\frac{2}{5}}
$$

Using (14), we follow the line of reasoning from the previous section to estimate $C_{2}^{P}$ as

$$
\begin{aligned}
1-e^{-9 C_{2}^{P}} & =\frac{X_{0}^{P}\left(\overline{\dot{\gamma}}=9 \mathrm{~s}^{-1}\right)}{X_{0}^{P}(\bar{\gamma}=\infty)} \\
& =0.65^{\frac{5}{2}} \approx 0.34 \Rightarrow C_{2}^{P} \approx 0.05 \mathrm{~s} .
\end{aligned}
$$

We can now equate (14) with the empirical result (4) and obtain

$$
\begin{aligned}
W^{P} & =D^{\frac{3}{5}}\left[\frac{C_{1}\left(1-e^{-0.05 \bar{\gamma}}\right) \times 15 \pi\left(1-H_{D}\right)}{256}\right]^{\frac{2}{5}} \\
& =K_{2}\left(1-e^{-0.05 \bar{\gamma}}\right)^{\frac{2}{5}}\left(1-H_{D}\right)^{\frac{2}{5}} D^{\frac{3}{5}},
\end{aligned}
$$

where $K_{2}=\left(15 \pi C_{1} / 256\right)^{\frac{2}{5}} \approx 0.51$. Thus, we have obtained a functional relationship between CFL width $W^{P}$ and discharge haematocrit $H_{D}$ (dependent on vessel diameter $D$ ) that should provide a good approximation for a fully established CFL whenever the velocity profile is close to parabolic and $W^{P} \ll D$. Using the parameter values from [27] (as discussed in the previous section), we conclude that $W^{P} \approx 2.2$.

As expected, the value of the CFL width predicted by the lattice Boltzmann simulation $(1.9 \mu \mathrm{m})$ is intermediate between those for the uniform $(1.24 \mu \mathrm{m})$ and parabolic $(2.2 \mu \mathrm{m})$ velocity profiles. Further, for shear rates with values as low as those used in the lattice Boltzmann simulation from [27] $\left(\bar{\gamma} \approx 9 \mathrm{~s}^{-1}\right)$, it has been shown that RBC aggregation has a significant effect on the (established) CFL width and inclusion of these effects in DPD simulations leads to a significantly thicker CFL [see Fig. 6(c)] [8]. As these effects are not included in [27], it is possible that in vivo values of the CFL width will be close to the value estimated for the parabolic velocity profile.

\section{Blunt velocity profiles}

In the core region, the presence of RBCs increases the viscosity of the blood, leading to blunt (flattened) velocity profiles. At low (tube) haematocrit levels, the profiles remain approximately parabolic, but they become increasingly blunt with increasing haematocrit (see Fig. 7). Moreover, flattening in the core region is more pronounced for thinner vessels (see, also, [14]). For a vessel of length $L$ experiencing a pressure difference $\Delta p$ between its two ends, one often rearranges the Poiseuille law (see [9]) to define the apparent viscosity of blood as

$$
\mu_{\mathrm{app}}=\frac{\pi}{128} \frac{\Delta p D^{4}}{L Q} .
$$

As RBCs migrate away from the vessel wall, the apparent viscosity depends on the vessel diameter and the discharge haematocrit in a nontrivial manner, which is now known as the Fåhraeus-Lindqvist effect [30]. We further define the relative apparent viscosity as $\mu_{\text {rel }}\left(D, H_{D}\right)=\mu_{\text {app }}\left(D, H_{D}\right) / \mu_{p}$, where $\mu_{p}$ is the plasma viscosity. In this work, we employ empirical relationships for $\mu_{\text {rel }}\left(D, H_{D}\right)$ deduced from in vivo data in [31]. Following [9], we then deduce that the blunt velocity profile $v^{B}(r)$ takes the form

$$
v^{B}(r)=\frac{32 Q}{\pi D^{4}} \mu_{\text {rel }}\left(D, H_{D}\right) \begin{cases}\left(\frac{D}{2}\right)^{2}-\left(\frac{D}{2}-W\right)^{2}+\frac{\mu_{p}}{\mu_{c}}\left[\left(\frac{D}{2}-W\right)^{2}-r^{2}\right] & \text { for } r \leqslant D / 2-W \\ \left(\frac{D}{2}\right)^{2}-r^{2} & \text { for } r \geqslant D / 2-W,\end{cases}
$$

where $\mu_{c}$ is the viscosity of the core region. Using the arguments from the previous section, it is straightforward to derive the following approximation for the minimum flow fraction:

$$
X_{0}^{B} \approx \mu_{\mathrm{rel}}\left(D, H_{D}\right) \frac{256}{15 \pi}\left(\frac{W^{B}}{D}\right)^{\frac{5}{2}} .
$$


We remark that this approximation for $X_{0}^{B}$ does not depend on $\mu_{c}$ because we only integrate the velocity field over the CFL $(r \geqslant D / 2-W)$. Equating Eqs. (4) and (19), we conclude

$$
W^{B}=K_{2}\left(1-e^{-0.05 \bar{\gamma}}\right)^{\frac{2}{5}} \frac{\left(1-H_{D}\right)^{\frac{2}{5}} D^{\frac{3}{5}}}{\mu_{\text {rel }}^{2 / 5}\left(D, H_{D}\right)},
$$

where the definition of the constant $K_{2}$ is identical to that from the previous section.

To summarize this section, in Table II, we list the formulas for the CFL width $W$ for the three models together with the corresponding assumptions on the flow velocity profile.

\section{COMPARISON WITH IN VIVO DATA}

Here, we compare the predictions of our models with the existing in vivo experiments on (fully established) CFL width from Refs. [10,11]. In these studies, the shear rates experienced by the vessels are high enough to justify neglecting the term $e^{-C_{2} \bar{\gamma}}$ in Eq. (4). We converted tube haematocrits from these works to discharge haematocrits using formulas from [32], thus accounting for the so-called Fåhraeus effect [33].

In [10], the authors studied the dependence of the CFL width $W$ on vessel diameter $D$ for a range of values of the tube haematocrit $\left(H_{T}=0.08,0.16,0.30\right.$, and 0.45$)$. The results are presented in Fig. 3 and, up to a small error, are within the ranges delimited by the uniform (from below) and parabolic (from above) models, except for very low-haematocrit values. As expected, the parabolic and uniform models provide better fits to the low-haematocrit and high-haematocrit data, respectively. The blunt-profile model predictions are typically intermediate between those for the uniform and parabolic models, and seem to capture the trends well, especially for $H_{T}=0.30$. However, for other values of the tube haematocrit, the discrepancies are significant. We will discuss possible sources of these (and other) errors in the next section. Next, we reconstruct the results from [10] displaying the dependence of $W$ on $H_{T}$ for comparison with our models; see Fig. 4. We again confirm that the parabolic model represents a good approximation for low-haematocrit values, the uniform model for high-haematocrit values, and the experimental data typically lie within the ranges delineated by the parabolic and uniform models, except for (extremely) low-haematocrit values. Most importantly, we note that the blunt-velocity profile model captures the dependence of the CFL width on haematocrit more accurately than either the parabolic or the uniform-profile model. In Fig. 5, we compare our model predictions with more recent in vivo data, obtained for tube haematocrit values of $H_{T} \approx 0.42$ [11]. If we neglect very thin vessels, then the experimental data fall within the ranges predicted by the parabolic- and uniform-profile models. Moreover, the blunt-velocity profile model accurately predicts the

TABLE II. Functional relationships for the CFL width $W$ for uniform, parabolic, and blunt velocity profiles.

\begin{tabular}{lll}
\hline \hline Flow profile & \multicolumn{1}{c}{ Formula for $W$} & Validity \\
\hline uniform & $K_{1}\left[\left(1-e^{-0.08 \bar{\gamma}}\right)\left(1-H_{D}\right)\right]^{\frac{2}{3}} D^{\frac{1}{3}}$ & high $H_{D}$ \\
parabolic & $K_{2}\left[\left(1-e^{-0.05 \bar{\gamma}}\right)\left(1-H_{D}\right)\right]^{\frac{2}{5}} D^{\frac{3}{5}}$ & low $H_{D}$ \\
blunt & $K_{2}\left[\frac{\left(1-e^{-0.05 \bar{\gamma}}\right)\left(1-H_{D}\right)}{\mu_{\mathrm{rel}}\left(D, H_{D}\right)}\right]^{\frac{2}{5}} D^{\frac{3}{5}}$ & any $H_{D}$ \\
\hline \hline
\end{tabular}

dependence of the CFL width $(W)$ on the vessel diameter $(D)$ and again proves to be superior to the other models. Recalling that we neglect the term $e^{-C_{2} \dot{\gamma}}$ in this section, this model only contains one free parameter $K_{2}=\left(15 \pi C_{1} / 256\right)^{2 / 5}$ [see (20)] where we estimated $C_{1} \approx 1$ in Sec. II A, which gave $K_{2} \approx$ 0.51 . By plotting the predictions of the blunt-velocity profile model for varying $K_{2}$, we find that the model prediction using twice the default value overestimates in a proportionate manner, while the prediction using half the default value underestimates the in vivo data (see Fig. 5). The model is thus sensitive to this parameter and our estimate for $C_{1}$ leads to results which are in good agreement with the in vivo data.

The tube haematocrit value from [11] is closest to the value 0.45 from [10]. We note that while the data from [11] are almost midway between the predictions for the uniform- and parabolic-profile models, the data from [10] lie below the predictions of the uniform-profile model. This discrepancy may be due to differences in the shear rates used in [10] and [11]. Indeed, if the shear rates in [10] are lower than those in [11], then the model predictions for [10] would lie slightly below those shown in Fig. 3.

\section{DISCUSSION}

We have derived models for the dependence of the fully established CFL width $(W)$ on vessel diameter $(D)$, discharge haematocrit $\left(H_{D}\right)$, and the average shear rate $(\bar{\gamma})$. To achieve this, we first extended the existing empirical fit for the minimum flow fraction from [23] to account for the average shear rate and then compared this expression with new expressions for this fraction, derived under the simplifying assumptions SA.1-SA.8 and based on simple flow-separation ideas, for

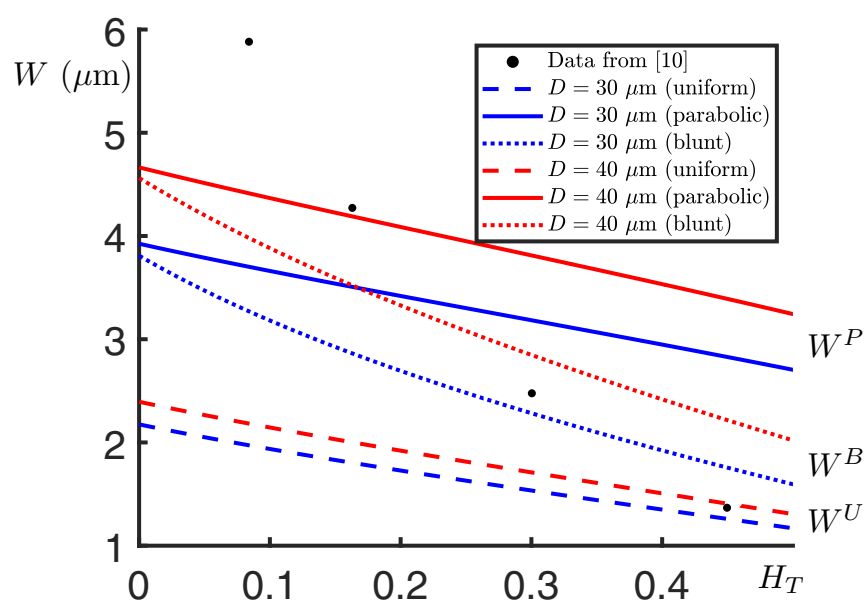

FIG. 4. Fully established CFL width $(W)$ as a function of vessel haematocrit $\left(H_{T}\right)$ for fixed values of the vessel diameter $D$. Data points represent in vivo averages from [10], the solid lines correspond to predictions from the parabolic model (17), dotted lines correspond to the blunt-profile model (20), and dashed lines to the uniform model (12), using $D=30 \mu \mathrm{m}$ (blue curves) and $D=40 \mu \mathrm{m}$ (red curves). 


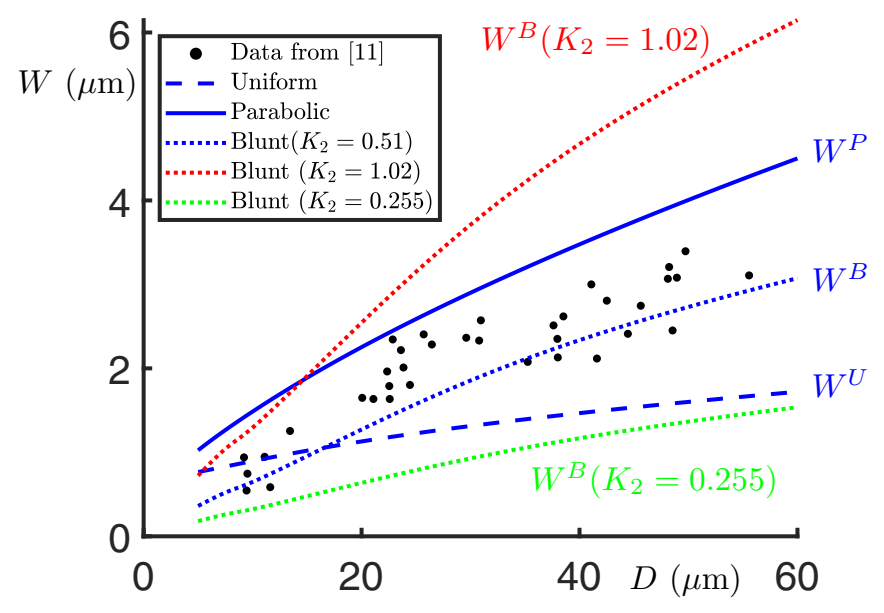

FIG. 5. Fully established CFL width $(W)$ as a function of vessel diameter for tube haematocrit $H_{T}=0.42$. Data points represent in vivo measurements from [11], the solid blue line gives predictions for the parabolic model (17), the dotted blue line for the blunt-profile model (20), and the dashed blue line for the uniform model (12). As anticipated, the data lie between the predictions of the parabolic and the uniform model, except those for very thin vessels. Overall, the blunt-velocity profile model most closely captures the behavior. By plotting the predictions of this model for twice (dotted red line) and half (dotted green line) the default value of $K_{2}(0.51)$, we show that the model is sensitive to $K_{2}=\left(15 \pi C_{1} / 256\right)^{2 / 5}$ and confirm the validity of our estimate for $C_{1}$ from Sec. II A.

uniform, parabolic, and blunt-velocity profiles upstream of the relevant bifurcation. As expected, predictions of the parabolicprofile model are in good agreement with the in vivo data in the low-haematocrit range, while the uniform-profile model captures the high-haematocrit data well. The blunt-profile model most accurately predicts the CFL width across a range of values of the vessel diameter and (especially) discharge haematocrits. Moreover, the scatter of existing in vivo data - as discussed in [12] and [13] — could be due to vessels experiencing different average shear rates, which means that our simple models might provide reliable predictions for in vivo behavior. More, and better controlled, experimental data are needed to more accurately assess the range of validity for the three models, especially with regards to the dependence on the mean shear rate. Our work also serves as a bridge between the two subfields of blood rheology research-the HS models and the DPD studies of phase separation within a single vessel-thus providing further consistency checks across state-of-the-art scientific theories.

Even with the blunt-profile model providing the most accurate knowledge of cross-sectional velocity profile, we see that discrepancies with the in vivo data can be significant. Such errors may be attributed to a combination of measurement errors and modeling simplifications.

\section{A. Measurement errors}

A key factor when considering measurement errors is the dynamic nature of blood rheology: the CFL width exhibits significant spatiotemporal variations (such variations are neglected in this work; see SA.2), which makes a precise definition of CFL width challenging and the corresponding measurements noisy. Moreover, predictions of DPD simulations from [12] and [13] were shown to differ from the in vivo data; a possible reason for this discrepancy is the presence of an endothelial surface layer (ESL). This layer has been shown to have a major impact on blood rheology. While the in vivo data in [11] accounted for the ESL thickness, it is unclear whether this was done in [10]. Given the estimated thickness of this layer $(0.4-0.5 \mu \mathrm{m}$ [12]), the data from [10] could significantly underestimate the CFL width. Our models should account for the ESL, albeit implicitly, as the fit for $X_{0}$ from (2) was obtained by fitting to in vivo data. Finally, we note that the spatial resolution in [11] was reported to be around $0.4 \mu \mathrm{m}$, which only adds to the uncertainty in the measurements.

\section{B. Range of validity of modeling simplifications and future model development}

To conclude, we revisit some of the key modeling assumptions used in this work and discuss the extent to which they may contribute to discrepancies between the experiments and the models. We also consider the parameter ranges for which these approximations are valid and outline possible ways to reduce errors associated with these simplifications.

\section{Validity of neglecting RBC size (SA.7)}

Consider a diverging bifurcation and a surface that partitions the parent vessel into two subregions, so that flow from one subregion enters one daughter vessel and flow from the second subregion enters the other daughter vessel. Let us assume further that one of the two daughter branches receives no RBCs and let us denote the corresponding subregion as subregion A. As a first approximation, RBCs in the parent vessel follow the streamline going through their centers of mass (SA.6). As a result, it is possible that a small part of an RBC may lie in subregion A. In such cases, the minimum flow fraction $X_{0}$ might be slightly greater than that calculated in this work. This might induce significant errors in the model predictions, particularly if RBCs are comparable in size with the vessel diameter (i.e., for very thin vessels). Future model extensions could incorporate the RBC diameter as a new parameter. However, Figs. 3 and 5 show that our model continues to perform well for very thin vessels.

\section{Validity of assuming $W / D \ll 1$ (SA.8)}

Our models yield the largest errors when discharge haematocrit levels are low and it is noteworthy that such (haemodiluted) vessels are abundant in tumor microenvironments. A likely explanation for this is that under low-haematocrit conditions, CFL width is large compared to the vessel diameter (see Fig. 7). In such cases, errors arising from truncating the Taylor series expansion may not be negligible. To correct for this, we might retain higher-order terms in the Taylor series expansions and, by following the same steps as before, establish whether the resulting CFL widths are in better agreement with the in vivo data under low-haematocrit conditions. However, the retention of higher-order terms will make the derivation of an explicit expression for the CFL width $W$ more challenging, as it would require solution of a polynomial equation of degree 5 (uniform model) or higher (parabolicand blunt-profile models).

\section{Validity of the empirical fits defined by Equations (1) and (2)}

We note that Eqs. (1) and (2) are empirical fits and not based on mechanistic considerations. In practice, the 
dependence of $X_{0}$ on $H_{D}$ and $D$ is likely to be more involved. Furthermore, relationships (1) and (2) were obtained by fitting to experimental data for vessels with diameters less than $50 \mu \mathrm{m}$ [21]. In vivo measurements of CFL thickness were taken from vessels with diameters in similar ranges [10,11]. As we expect approximations (SA.8) $(\delta \ll 1)$ and (SA.7) (the negligibility of RBC size) to hold for thicker vessels, it seems worthwhile to compare our model with in vivo measurements for microvessels larger than $50 \mu \mathrm{m}$ in diameter. Unfortunately, we were unable to find such data in the literature.

In summary, given its limitations and the need for further experimental testing, the work presented in this article represents an important first step to obtaining models of microvascular blood rheology that are simultaneously accurate and simple enough to be easily upscaled to larger spatial scales. Particularly for small vessels, the applicability of purely mechanistic models is severely limited by the computational complexity of capillary beds and the inappropriateness of standard continuum approaches [34]; in such situations, reduced-order models, of the type presented here, represent a promising alternative.

\section{ACKNOWLEDGMENTS}

We thank M.O. Bernabeu for many helpful discussions. This work was supported by Cancer Research UK (CRUK) Grants No. C5255/A18085 and No. C5255/A15935.

\section{APPENDIX A: RELEVANT FIGURES REPRODUCED FROM THE LITERATURE}

For completeness, we reproduce, from the literature, figures of relevance to the model development presented in the

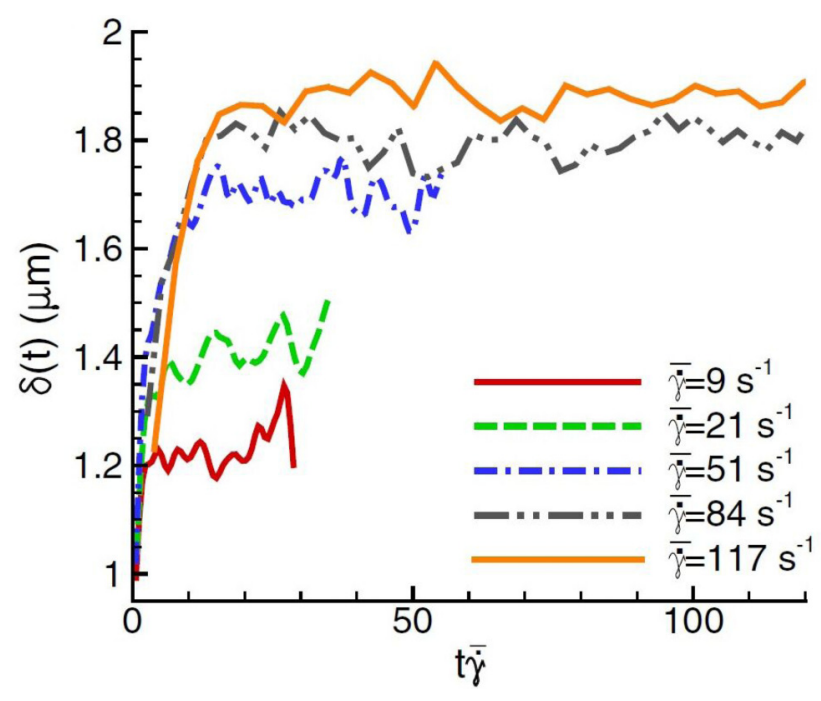

(a)

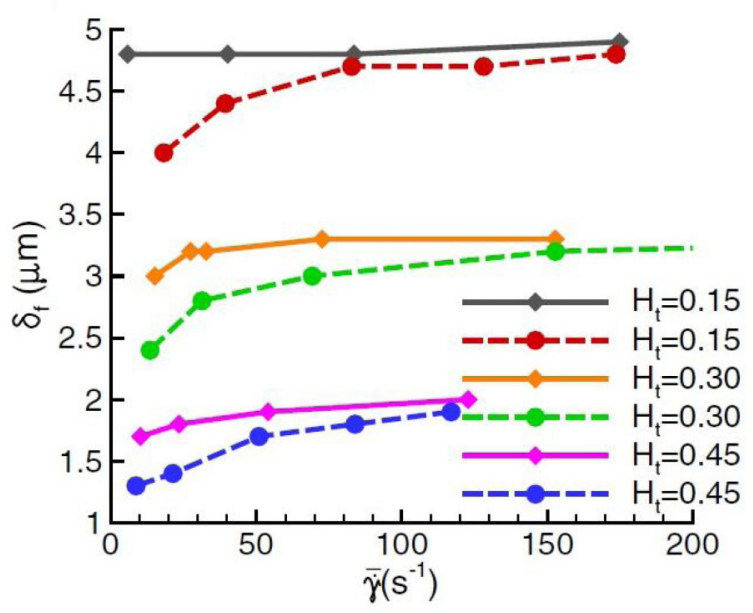

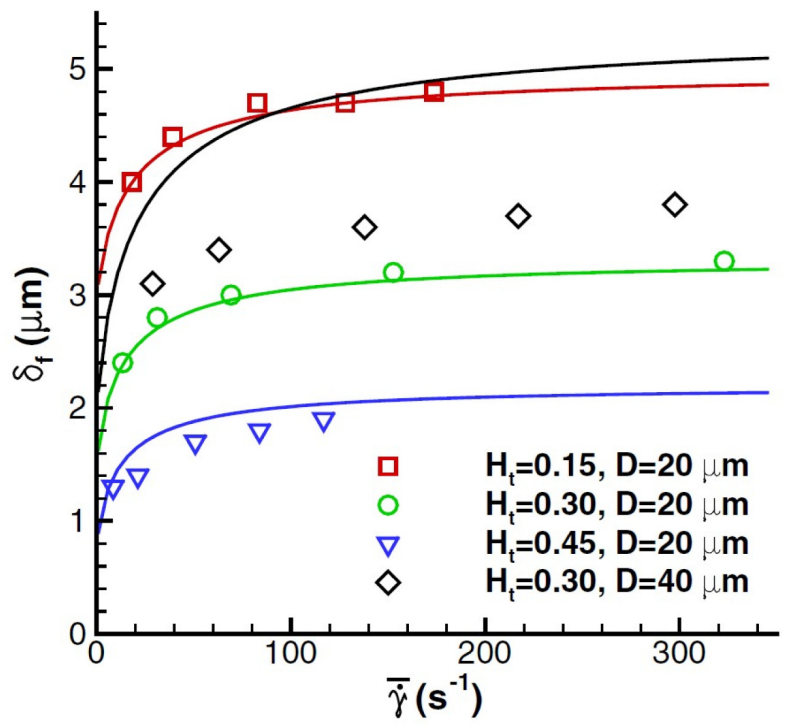

(b)

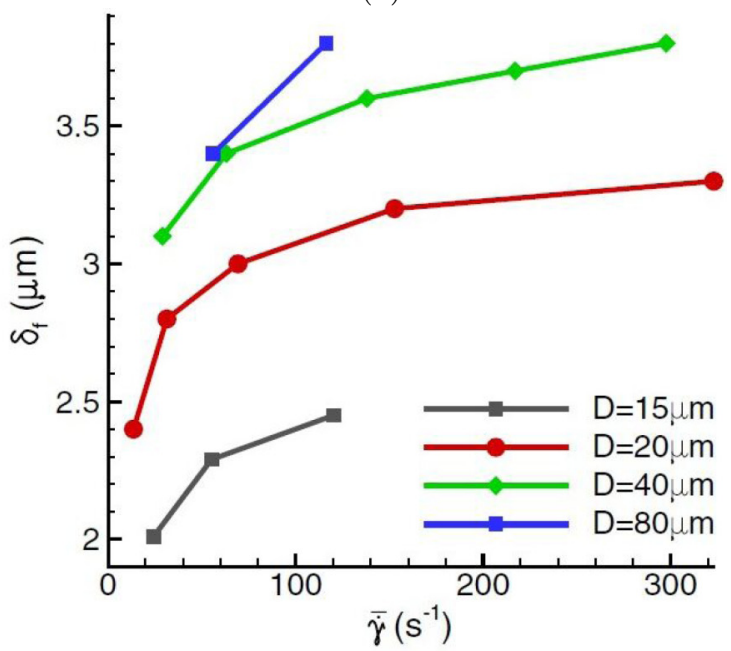

(c)

FIG. 6. Reproduction of key figures 3(a), 8(b), and 7(c) from [8] (with permission). (a) Starting from a dispersed cross-sectional distribution of RBCs, the time evolution of CFL width [here denoted $\delta(t)$ ] as dependent on the mean shear rate $\bar{\gamma}$ was studied using DPD. (b), (c) Then, the dependence of the converged (i.e., fully established) CFL width (here denoted $\delta_{f}$ ) on the mean shear rate $\bar{\gamma}$, vessel diameter $D$, and tube haematocrit $H_{t}$ was examined. Note that the dashed lines in (c) show simulations that do not incorporate the effect of RBC aggregation (whereas the solid lines do). 

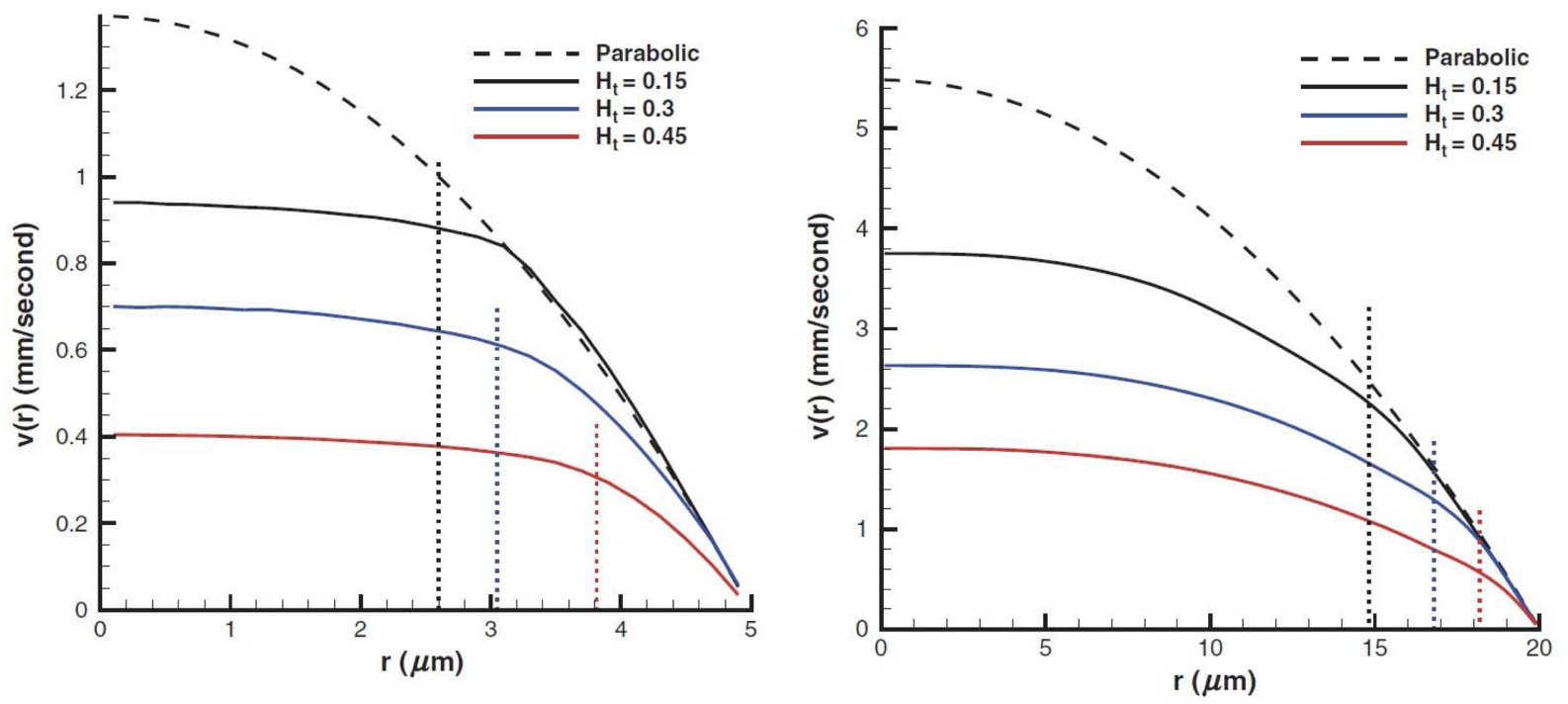

FIG. 7. Typical velocity $v$ as a function of radial distance from the vessel centerline $r$ as dependent on tube haematocrit $H_{\mathrm{t}}$ and vessel diameter $D$ reproduced from [12] (Fig. 2 in the reference; with permission). The subfigure on the left uses $D=10 \mu \mathrm{m}$ and that on the right uses $D=40 \mu \mathrm{m}$.

main body of this paper. These figures relate to the DPD simulations of CFL width from [8] (Fig. 6) and cross-sectional velocity profiles from [12] (Fig. 7).

\section{APPENDIX B: MODEL PARAMETRIZATIONS FROM [22] AND [23]}

Based on experimental observations, Pries et al. [22] proposed the following haematocrit splitting model:

$$
\operatorname{logit}\left(F Q_{E}\right)=A+B \operatorname{logit}\left(\frac{F Q_{B}-X_{0}}{1-2 X_{0}}\right),
$$

where $F Q_{E}$ is the volume fraction of RBCs entering one branch, $F Q_{B}$ is the fraction of the total blood flow entering that branch, $X_{0}$ is the minimum flow fraction in either branch for RBCs to enter that branch, and $\operatorname{logit}(x)=\ln [x /(1-x)]$. The $\left(1-2 X_{0}\right)$ term reflects the fact that the CFL exists for both daughter vessels. The dimensionless parameters $A, B$, and $X_{0}$ in the model from [22] depend on the diameters $D$ of the parent and both children vessels (denoted by subscripts $\alpha$ and $\beta$, respectively), and the discharge haematocrit $H_{D}$ in the parent vessel, in the following way:

$$
\begin{aligned}
& A=-6.96 \ln \left(\frac{D_{\alpha}}{D_{\beta}}\right) / D, \\
& B=1+6.98\left(\frac{1-H_{D}}{D}\right),
\end{aligned}
$$

and

$$
X_{0}=\frac{0.4}{D} .
$$

To obtain a better approximation for extreme combinations of parameter values, the dependences of $A$ and $X_{0}$ on model parameters from [22] were later modified in [23] as follows:

$$
A=-13.29\left[\left(D_{\alpha}^{2} / D_{\beta}^{2}-1\right) /\left(D_{\alpha}^{2} / D_{\beta}^{2}+1\right)\right]\left(1-H_{D}\right) / D
$$

and

$$
X_{0}=0.964\left(1-H_{D}\right) / D
$$

We use both Eqs. (B4) and (B5) in this work.
[1] D. Treacher and R. Leach, Bmj 317, 1302 (1998).

[2] D. F. Wilson, Am. J. Physiol.: Heart Circ. Physiol. 294, H11 (2008).

[3] L. H. Gray, A. D. Conger, M. Ebert, S. Hornsey, and O. C. A. Scott, Br. J. Radiol. 26, 638 (1953).

[4] J. A. Bertout, S. A. Patel, and M. C. Simon, Nat. Rev. Cancer 8, 967 (2008).

[5] S. Rockwell, I. T. Dobrucki, E. Y. Kim, S. T. Marrison, and V. T. Vu, Curr. Molec. Med. 9, 442 (2009).

[6] K. Graham and E. Unger, Intl. J. Nanomed. 13, 6049 (2018).

[7] B. S. Sørensen and M. R. Horsman, Frontiers Oncol. 10, 562 (2020).
[8] D. Katanov, G. Gompper, and D. A. Fedosov, Microvasc. Res. 99, 57 (2015).

[9] T. W. Secomb, Annu. Rev. Fluid Mech 49, 443 (2017).

[10] N. Tateishi, Y. Suzuki, M. Soutani, and N. Maeda, J. Biomech. 27, 1119 (1994).

[11] S. Kim, R. L. Kong, A. S. Popel, M. Intaglietta, and P. C. Johnson, Am. J. Physiol.: Heart Circ. Physiol. 293, H1526 (2007).

[12] D. A. Fedosov, B. Caswell, A. S. Popel, and G. E. M. Karniadakis, Microcirculation 17, 615 (2010).

[13] W. Pan, D. A. Fedosov, B. Caswell, and G. E. Karniadakis, Microvasc. Res. 82, 163 (2011). 
[14] H. Lei, D. A. Fedosov, B. Caswell, and G. E. Karniadakis, J. Fluid Mech. 722, 214 (2013).

[15] V. W. Azizi Tarksalooyeh, G. Závodszky, B. J. M. van Rooij, and A. G. Hoekstra, Comput. Fluids 172, 312 (2018).

[16] B. Van Rooij, G. Závodszky, V. Azizi Tarksalooyeh, and A. Hoekstra, J. R. Soc. Interface 16, 20190148 (2019).

[17] Z. Liu, J. R. Clausen, R. R. Rao, and C. K. Aidun, Phys. Fluids 31, 081903 (2019).

[18] Z. L. Liu, J. R. Clausen, J. L. Wagner, K. S. Butler, D. S. Bolintineanu, J. B. Lechman, R. R. Rao, and C. K. Aidun, Phys. Rev. E 102, 013310 (2020).

[19] M. Sharan and A. S. Popel, Biorheology 38, 415 (2001).

[20] P. Bagchi, Biophys. J. 92, 1858 (2007).

[21] A. Pries, K. Ley, M. Claassen, and P. Gaehtgens, Microvasc. Res. 38, 81 (1989).

[22] A. R. Pries, T. W. Secomb, P. Gaehtgens, and J. F. Gross, Circ. Res. 67, 826 (1990).

[23] A. R. Pries and T. W. Secomb, Am. J. Physiol. 289, H2657 (2005).
[24] Y.-C. Fung, Biomechanics: Circulation, 2nd ed. (Springer, New York, 1997).

[25] I. G. Gould and A. A. Linninger, Microcirculation 22, 1 (2015).

[26] J. Yang, S. S. Yoo, and T.-R. Lee, Phys. Rev. E 95, 040401(R) (2017).

[27] M. O. Bernabeu, J. Köry, J. A. Grogan, B. Markelc, A. Beardo, M. d'Avezac, R. Enjalbert, J. Kaeppler, N. Daly, J. Hetherington et al., Proc. Natl. Acad. Sci. 117, 27811 (2020).

[28] K. Ley, A. R. Pries, and P. Gaehtgens, Pflügers Archiv. Europ. J. Physiol. 412, 93 (1988).

[29] T. W. Secomb, Comprehens. Physiol. 6, 975 (2016).

[30] R. Fahraeus and T. Lindqvist, Am. J. Physiol. Legacy Content 96, 562 (1931).

[31] A. R. Pries, T. W. Secomb, T. Gessner, M. B. Sperandio, J. F. Gross, and P. Gaehtgens, Circ. Res. 75, 904 (1994).

[32] A. R. Pries, D. Neuhaus, and P. Gaehtgens, Am. J. Physiol. 263, H1770 (1992).

[33] R. Fåhraeus, Klinische Wochenschrift 7, 100 (1928).

[34] R. J. Shipley, A. F. Smith, P. W. Sweeney, A. R. Pries, and T. W. Secomb, Math. Med. Biol.: J. IMA 37, 40 (2020). 